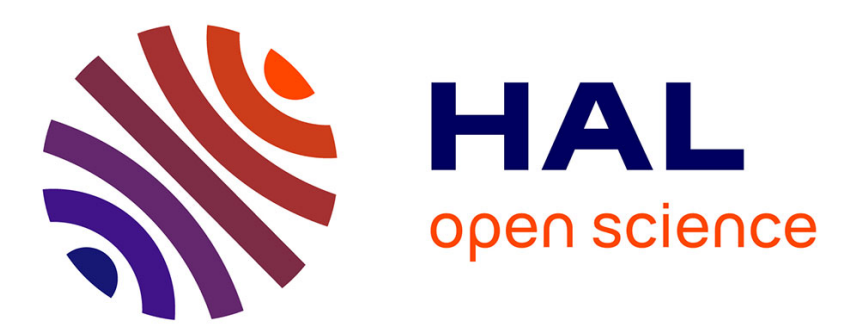

\title{
Automatic Fault Detection for 2D Seismic Data Based on the Seismic Coherence of Mutative Scale Analysis Window
}

Wenli Zheng, Jinwen Ma

\section{- To cite this version:}

Wenli Zheng, Jinwen Ma. Automatic Fault Detection for 2D Seismic Data Based on the Seismic Coherence of Mutative Scale Analysis Window. 2nd International Conference on Intelligence Science (ICIS), Nov 2018, Beijing, China. pp.391-400, 10.1007/978-3-030-01313-4_42 . hal-02118843

\section{HAL Id: hal-02118843 \\ https://hal.inria.fr/hal-02118843}

Submitted on 3 May 2019

HAL is a multi-disciplinary open access archive for the deposit and dissemination of scientific research documents, whether they are published or not. The documents may come from teaching and research institutions in France or abroad, or from public or private research centers.
L'archive ouverte pluridisciplinaire HAL, est destinée au dépôt et à la diffusion de documents scientifiques de niveau recherche, publiés ou non, émanant des établissements d'enseignement et de recherche français ou étrangers, des laboratoires publics ou privés.

\section{(c)(1)}

Distributed under a Creative Commons Attribution| 4.0 International License 


\title{
Automatic Fault Detection for 2D Seismic Data Based on the Seismic Coherence of Mutative Scale Analysis Window
}

\author{
Wenli Zheng ${ }^{1,2}$ and Jinwen $\mathrm{Ma}^{2}$ \\ 1 School of Science, Xi'an Shiyou University, Xi'an, Shaanxi Province, 710065, P. R. \\ China \\ wlzheng@xsyu.edu.cn \\ 2 School of Mathematical Sciences \& LMAM, Peking University, Beijing, 100871, P. \\ R. China \\ jwma@math.pku.edu.cn
}

\begin{abstract}
Fault detection is a very challenging problem on seismic interpretation. In fact, the process of fault detection contains seismic attribute extraction, seismic attribute enhancement and fault line detection. It is clear that the extracted seismic attributes are key to the fault detection process. Traditionally, as an important seismic attribute, the seismic coherence is generally employed in the fault detection process, but the size of the analysis window often effects the value of the seismic coherence, and there is a trade-off between the vertical resolution and the lateral resolution. In order to overcome this problem, we propose a new kind of seismic coherence with a mutative scale analysis window, and utilize it to locate the fault lines. It is demonstrated by the experimental results that our proposed seismic coherence is more suitable for the fault line detection in comparison with the traditional seismic coherence.
\end{abstract}

Keywords: Fault detection, Seismic attribute, Seismic coherence, Mutative scale, Analysis window.

\section{Introduction}

For detecting the positions of energy resources under a specific ground, it is often necessary to collect and analyze the seismic data generated from a seismic exploration work which contains seismic data collection, seismic data processing and seismic interpretation. After collected and calibrated, a seismic data volume can be formed corresponding to the space under the ground. Finally we will analyze the seismic data by some theory and technology to obtain underground information.

Fault detection is a very challenging problem in the seismic interpretation within a potentially enormous seismic volume. Geological faults are important since they are often associated with the formation of subsurface traps in which petroleum might accumulate. The process of automatic fault detection contains seismic attribute extraction, seismic attribute enhancement, and fault detection, 
where the seismic attributes extraction is a major step, and the seismic coherence is often used as one of seismic attributes in the fault detection process. Bahorich and Farmer [1] firstly introduced a classical normalized crosscorrelation to measure the continuity between neighboring windowed seismic traces, which was referred to as an attribute of coherence. Although this original coherence is computed efficiently, but lacks the robustness for noisy seismic data. Then Marfurt et al. [2] proposed a multi-trace semblance coherency, which estimates the coherency over multi seismic traces. In comparison with the original coherence, this one becomes stable in the emergence of noise, and improves the vertical resolution. However, by increasing seismic traces on computing the semblance coherence, the lateral resolution will decrease while the computational cost will increase. Then, Gersztenkorn and Marfurt [3] introduced an eigenstructure-based coherence which provides more stable seismic coherence but requires the computation of eigenvalues. In order to overcome the drawbacks of the eigenstructurebased coherence, Cohen et al. proposed two local discontinuity measure named seismic local structural entropy (LFE) [4] and normalized differential entropy (NDE) [5], respectively, being used to extract the faults. But these seismic attributes are dependent on the size of analysis windows which are selected by experience. In fact, the size of analysis window is rather difficult to be selected in practical faut detection. In this paper, we propose a new kind of seismic coherency measure with mutative scale analysis window to avoid the shortness of the above seismic attributes. In fact, this new seismic coherence can be effectively used for fault detection.

The rest of this paper is organized as follows. We begin to introduce the semblance coherence and the eigenstrcture-based coherence in Section 2. In Section 3 , we present a new seismic coherence. Section 4 summarizes the experimental results on a real seismic dataset. Finally, we conclude briefly in Section 5 .

\section{Conventional Seismic Coherence}

Before we introduce the semblance coherence and the eigenstrcture-based coherence, we firstly present a seismic slice and define an analysis window. The value of every points on a seismic slice is the seismic reflection amplitude of the seismic wave, shown in Figure 1. the horizontal direction is the inline or crossline, and the vertical direction is the time axis. when we estimate the seismic coherency value at a point, we should firstly design an analysis window at the center of this point, of which the size is $2 t+1 \times 2 K+1$, where $t$ and $K$ is set according to experts' experience. After that we present the semblance coherence and the eigenstrcture-based coherence, respectively.

\subsection{Semblance Coherence}

Let $C 2(i, j, \theta)$ be the semblance coherency value at the point $(i, j)$, and $\theta$ is the direction of the analysis window. The semblance coherency equation takes the 
following form.

$C 2(i, j, \theta)=\frac{\sum_{\tau=-t}^{t}\left\{\left(\sum_{k=-K}^{K} D(i+\tau+p k, j+k)\right)^{2}+\left(\sum_{k=-K}^{K} D^{H}(i+\tau+p k, j+k)\right)^{2}\right\}}{(2 K+1) \sum_{\tau=-t}^{t} \sum_{k=-K}^{K}(D(i+\tau, j+k))^{2}+\left(D^{H}(i+\tau, j+k)\right)^{2}}$

where $\mathrm{D}$ is the seismic slice and $D(i, j)$ represents the amplitude at the point $(i, j)$. Generally, $\theta$ is 0 .

\subsection{Eigenstrcture-based Coherence}

After introducing the semblance coherence, we show the eigenstrcture-based coherency calculation procedure. First, we extract all seismic data enclosed by the analysis window at the center of the point $(i, j)$, and get a matrix $S D_{i j}$ which is the following form.

$$
S D_{i j}=\left[\begin{array}{cccc}
d_{11} & d_{12} & \cdots & d_{1 J} \\
d_{21} & d_{22} & \cdots & d_{2 J} \\
\vdots & \vdots & \ddots & \vdots \\
d_{N 1} & d_{N 2} & \cdots & d_{N J}
\end{array}\right]
$$

where $N=2 t+1$ and $J=2 K+1$. Then construct a covariance matrix, denoted $C$, shown in Eq. 3

$$
C=S D_{i j}^{T} S D_{i j}=\left[\begin{array}{cccc}
\sum_{n=1}^{N} d_{n 1}^{2} & \sum_{n=1}^{N} d_{n 1} d_{n 2} & \cdots & \sum_{n=1}^{N} d_{n 1} d_{n J} \\
\sum_{n=1}^{N} d_{n 1} d_{n 2} & \sum_{n=1}^{N} d_{n 2}^{2} & \cdots & \sum_{n=1}^{N} d_{n 2} d_{n J} \\
\vdots & \vdots & \ddots & \vdots \\
\sum_{n=1}^{N} d_{n 1} d_{n J} & \sum_{n=1}^{N} d_{n 2} d_{n J} & \cdots & \sum_{n=1}^{N} d_{n J}^{2}
\end{array}\right]
$$

Finally, compute the eigenvalue of $C$, denoted as $\left(\lambda_{1}, \lambda_{2}, \cdots, \lambda_{J}\right)$, where $\lambda_{1} \geq$ $\lambda_{2} \geq \cdots \geq \lambda_{J}$. Therefore, The eigenstrcture-based coherency value takes the following form.

$$
C 3(i, j, \theta)=\frac{\lambda_{1}}{\sum_{n=1}^{J} \lambda_{n}},
$$

where $C 3(i, j, \theta)$ ranges form 0 to 1 .

Those two seismic coherence attributes are strongly affected by the size of the analysis window. If the length of the analysis window is larger, the more seismic traces are enclosed by the analysis window, and the lateral resolution will decrease. If the width of the analysis window is larger, the vertical resolution will decrease. There is a trade-off between the length and width of the analysis window, so we will propose a new attribute to estimate the seismic coherence.

\section{Proposed Seismic Coherence of Mutative Scale Analysis Window}

Here we introduce a new seismic coherence based on the semblance coherence and the eigenstrcture-based coherence. The traditional seismic coherency value 
changes as the size of the analysis window changes, so it is important to choose the size of analysis window. In order to overcome this problem, we propose a new attribute to estimate the seismic coherence with a series of analysis windows in different size. Next we take the eigenstrcture-based coherence as an example to present the variable scale eigenstrcture-based coherence.

Let $D$ be the seismic slice and $p=(i, j)$ be a point, then we compute the variable scale eigenstrcture-based coherency value at the point $p$. We initialize an analysis window, of which the size is $N \times K$ and the direction is $\theta$, and extract a dataset enclosed by the initial analysis window, then we estimates the eigenstrcture-based coherency value of $p$ by Eq. 4 and record this value. Next let $N=N+\Delta n$ and $K=K+\Delta w$ where $\Delta n$ and $\Delta w$ are constants, and repeat the above process. Finally, we obtain a series of the eigenstrcture-based coherency values by varying the size of the analysis window, and record these values in a matrix, denoted $G$. Because the eigenstrcture-based coherency value ranges from 0 to 1 , we name $G$ as a eigenstrcture-based coherency gray image of the point $p$. If this point $p$ located in different geological structure, its eigenstrcture-based coherency gray image has different texture. In order to measure those different texture, we introduce the Gray-level co-occurrence matrix(GLCM) of $G$ and use the average of the vertical and horizontal angular second moment(ASM)to measure the coherence of the point $p$, denoted $S C 3(i, j, \theta)$. Using the same method, we estimates the variable scale semblance coherency value of the point, $p$, denoted $S C 2(i, j, \theta)$. In order to take advantages of both variable scale coherence, we compute the average of $S C 2(i, j, \theta)$ and $S C 3(i, j, \theta)$, shown in Eq. 6

$$
S C(i, j, \theta)=\frac{S C 2(i, j, \theta)+S C 3(i, j, \theta)}{2} .
$$

Then let $\theta=\theta+\Delta \theta$, and get a sequence of $S C(i, j, \theta)$. Finally we define the new seismic coherence of the point $p$, which takes the following form.

$$
S C\left(i, j, \theta^{*}\right)=\arg \max _{\theta_{\min } \leq \theta \leq \theta_{\max }} S C(i, j, \theta) .
$$

So far, we have introduced our proposed attribute to estimate the new seismic coherence, and then we use this new seismic coherence as the seismic attribute to locate faults on a seismic slice.

\section{Fault Detection Through the Proposed Coherence}

After compute the new seismic coherence, we apply the Log-Gabor filter to enhance the new seismic coherence [7]. Finally we locate the fault lines on the seismic slice by the curve detection algorithm proposed by Carsten Streger [9].

The Log-Gabor filter is popular in the image processing [6] [8], because it considers the orientation information to save the image detail. The 2D Log-Gabor filter takes following form,

$$
G(f, \theta)=\exp \left(\frac{-\left(\log \left(f / f_{0}\right)\right)^{2}}{2 \log \left(\sigma_{f} / f_{0}\right)}\right) \exp \left(\frac{-\left(\log \left(\left\|\theta-\theta_{0}\right\|\right)^{2}\right.}{2 \sigma_{\theta}}\right)
$$


where $f$ and $\theta$ are parameters of the Log-Gabor filter. $f_{0}$ is the center frequency, and $\sigma_{f}$ is the width parameter for the of frequency. $\theta_{0}$ is the center orientation, and $\sigma_{\theta}$ is the width parameter for the orientation.

After the new coherence enhancement, we do the fault detection operation by the curve detection algorithm, named Steger's curve detection algorithm [9]. The Steger's curve detection algorithm is wildly used to detect the curves of satellite images and computed tomography images. Then we apply this new attribute on the new enhanced seismic coherence to detect the fault lines of seismic slices.

\section{Experimental Results}

We have presented the new coherence which is the average of the variable scale semblance coherence and the variable scale eigenstrcture-based coherence, and then we apply the Log-Gabor filter to improve the quality of our proposed seismic coherence. Finally the Steger curve detection algorithm is used to detect faults. In order to test the performance our proposed coherence, we estimate the coherence of a real data, named Qikou, of which the inline number is 2654, shown in Figure 1, and compare with the traditional semblance coherence, the traditional eigenstrcture-based coherence, and the local structure entropy(LSE)proposed by Cohen. Furthermore, we do the fault detection operation by the Steger curve algorithm on our proposed coherence, and compare with the manual labeled fault lines. Let the initial window size be $N \times K$, where $N$ ranges from 21 to 61 with

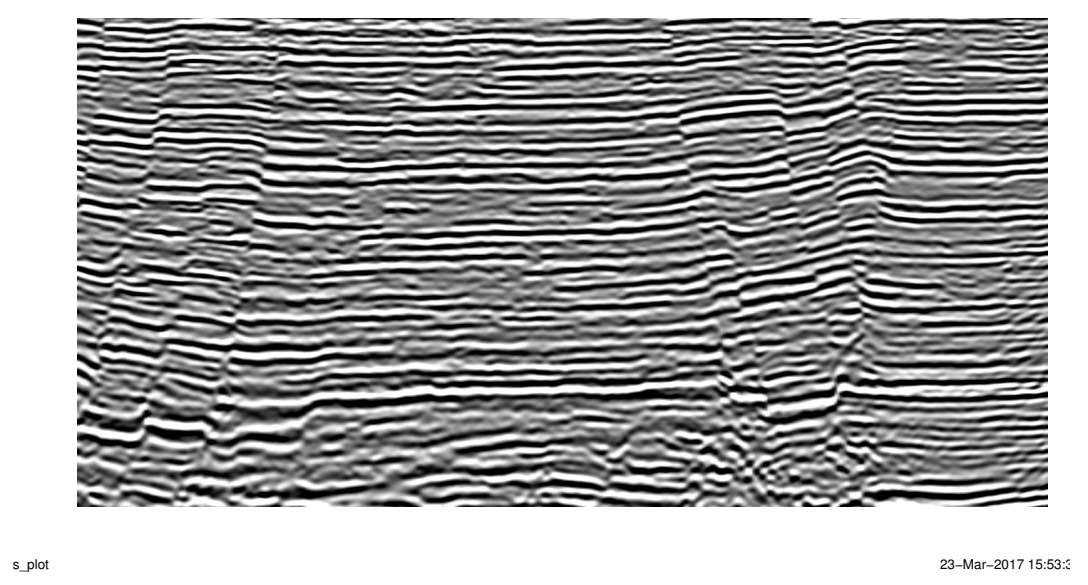

Fig. 1. The Qikou seismic slice is in the gray scale and the inline number is 2654

the step 4 , and $K$ ranges from 3 to 7 with the step 2 . the analysis window orientation varies from $-70^{\circ}$ to $70^{\circ}$ with the common difference $25^{\circ}$. Then we apply 
our propose seismic coherence on the Qikou seismic slice. Firstly we compare the traditional semblance coherence with the variable scale semblance coherence and show in Figure 2. On Figure 2, The positions pointed by red arrows are on the fault lines, but the traditional semblance coherency can not make them clear. The areas enclosed by the green circle are special geological structures, and the variable scale semblance coherency can distinct them. So the variable scale semblance coherency not only improve the vertical resolution but also increase the horizontal resolution, and it overcome weaknesses of the traditional semblance coherence. Then we compare the traditional eigenstructure-based coherence with the variable scale eigenstrcture-based coherence, shown in Figure 3, and we obtain that the variable scale eigenstrcture-based coherence is better than the traditional scale eigenstrcture-based coherence.

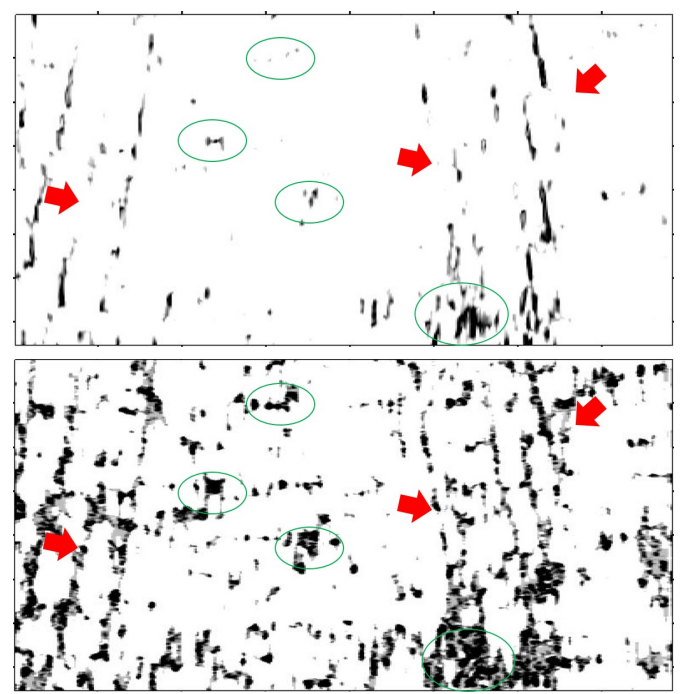

Fig. 2. The traditional semblance coherence(above)and the variable scale semblance coherence (below)

Following we compare the variable scale semblance coherence, the variable scale semblance coherence and the average of those two variable scale seismic coherence, shown in Figure 4. Although the variable scale semblance coherence and eigenstructure-based coherence are better than the traditional coherence, there are still details missing pointed by the blue and red arrow in Figure 4(above) and Figure 4(middle). Figure 4(below) is the average of those two variable scale seismic coherence, and we can see that the missing details in Figure 4(above) and Figure 4(middle) appear again. Additionally we compare the average of those two variable scale seismic coherence with LSE, shown in Figure 5. Therefore, we take the average of those two variable scale seismic coherence as a new seismic coherence. Finally apply the Log-Gabor filter on the new seismic co- 


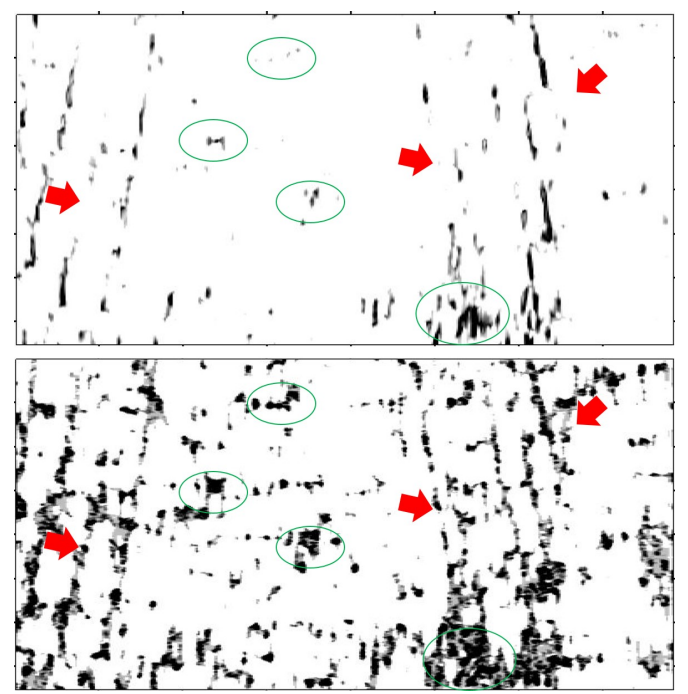

Fig. 3. The traditional eigenstructure-based coherence(above)and the variable scale eigenstructure-based coherence (below)

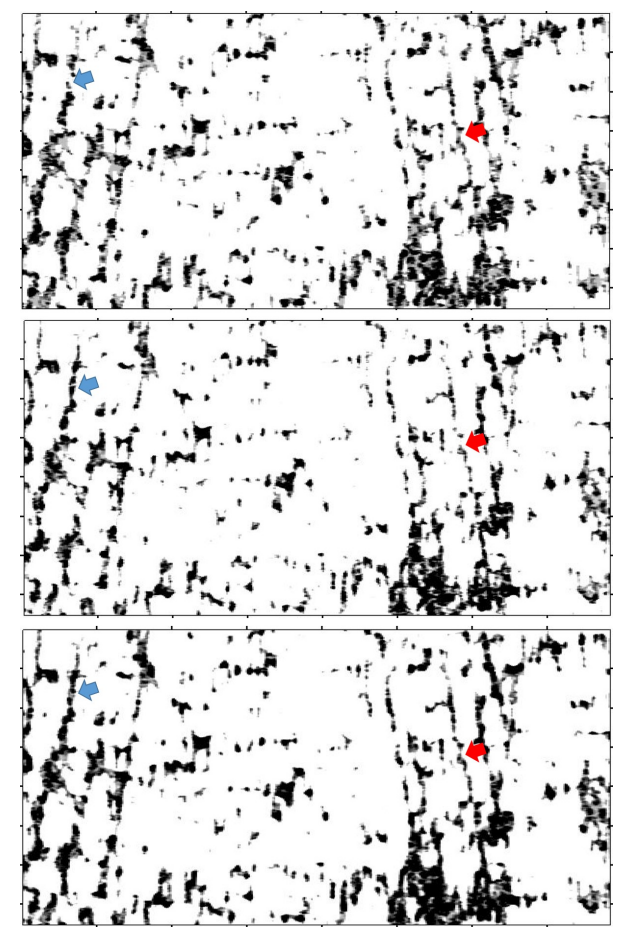

Fig. 4. The variable scale semblance coherence(above), the variable scale eigenstructure-based coherence(middle) and the average of the two variable scale coherence (below) 


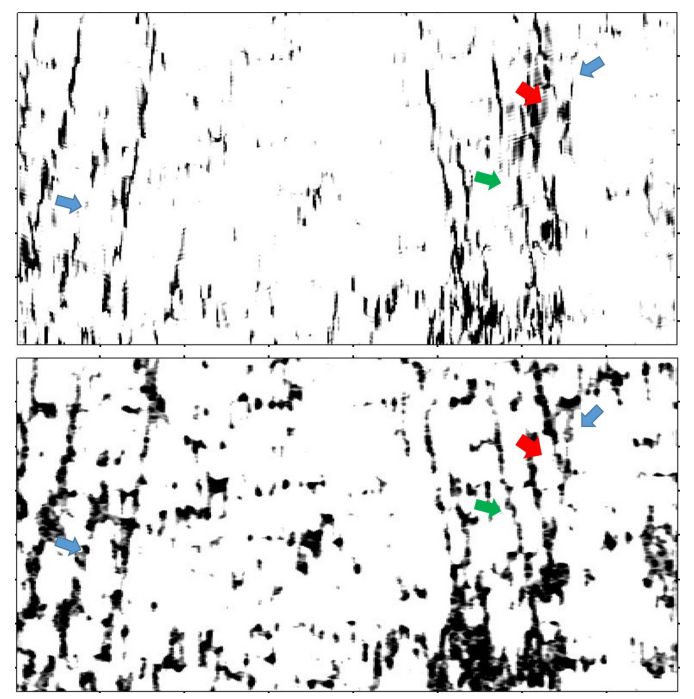

Fig. 5. LSE (above) and the average of the two types of the variable scale seismic coherence(below)

herence and use the Steger curve detection algorithm to locate fault lines of the Qikou seismic slice. The result is compared with the manual labeled fault lines, shown in Figure 6. Compared with the fault lines labeled manually, the fault lines detected by our proposed method is not totally same to the fault lines labeled manually, and the differences are pointed by yellow, red and blue arrows. but the performance of the Steger's curve detection algorithm is better than the Canny operator.

Further, we use the Steger curve detection algorithm on the semblance coherence, the eigenstructure-based coherence, the variable scale semblance coherence, the variable scale eigenstructure-based coherence, the average of those two variable scale seismic coherence and the LSE, respectively, shown in Figure 7. From the six images, we obtain that our proposed new seismic coherence is more suitable to be used as the seismic attributes making fault information clear.

\section{Conclusion}

We have proposed two types of seismic coherence, i.e., the semblance coherence and the eigenstructure-based coherence with mutative scale analysis window and fused the two seismic coherence attributes to form a new seismic coherence. LogGabor filter is further implemented on the new seismic coherence to improve its quality, and Steger's curve detection algorithm is used to locate the fault lines. It is demonstrated by the experimental results on the real seismic data that our proposed seismic coherence is better than the traditional seismic coherence and more suitable for fault detection. 

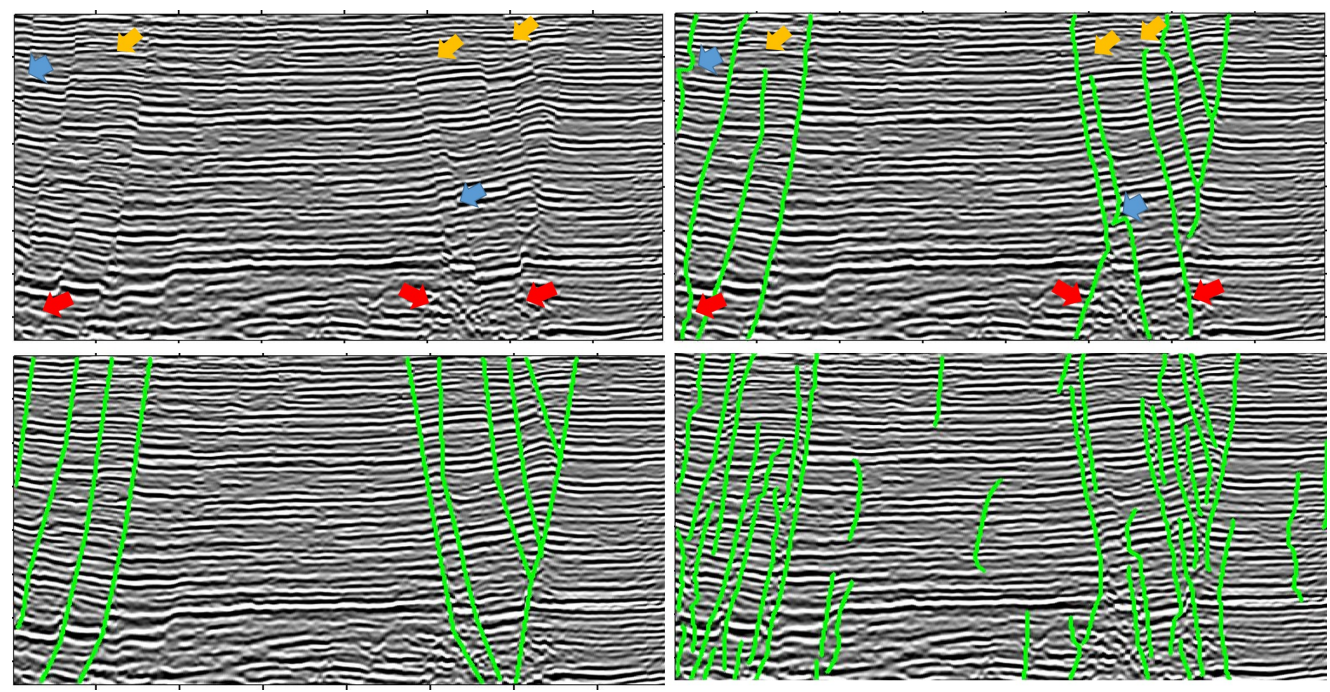

Fig. 6. From left to right and from up to down, the original seismic data of Qikou(a), the fault lines detected by the Steger's curve detection algorithm(b), the fault lines labeled manually(c), and the fault lines detected by the Canny operator(d)

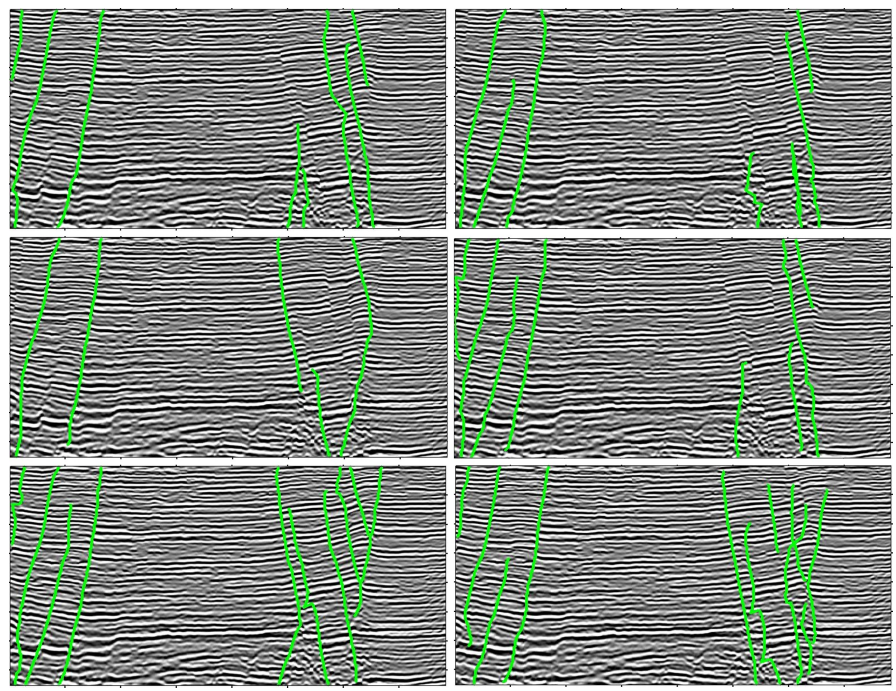

Fig. 7. From left to right and from up to down, the result of detection of the semblance coherence, the eigenstructure-based coherence, the variable scale semblance coherence, the variable scale eigenstructure-based coherence, the average of the two types of the variable scale seismic coherence and the LSE 


\section{Acknowledgment}

This work was supported by the BGP Inc., China National Petroleum Corporation.

\section{References}

1. Bahorich M, Farmer S.: 3D seismic discontinuity for faults and stratigraphic features: The coherence cube Seg Technical Program Expanded. 93-96 (1995).

2. Marfurt K J, Farmer S L, Bahorich M S, et al:. 3-D seismic attributes using a semblance-based coherency algorithm. Geophysics. 63(4):1150 (1998)

3. Gersztenkorn A, Marfurt K J.: Eigenstructure-based coherence computations as an aid to 3-D structural and stratigraphic mapping. Geophysics. 64(5):1468(1999)

4. Cohen I, Coifman R R.: Local discontinuity measures for 3-D seismic data. Geophysics. 67(6):1933-1945(2002)

5. Cohen I, Coult N, Vassiliou A A.: Detection and extraction of fault surfaces in 3D seismic data. Geophysics. 71(4):2006(2006)

6. Field D J.: Relations between the statistics of natural images and the response properties of cortical cells. Journal of the Optical Society of America A-optics Image Science \& Vision. 4(12):2379-2394(1987)

7. Yu Y.: Fault enhancement and visualization with 3D log-Gabor filter array. Seg Technical Program Expanded. 1960-1965(2016)

8. Wang W, Li J, Huang F, et al.: Design and implementation of Log-Gabor filter in fingerprint image enhancement. Pattern Recognition Letters, 29(3):301-308(2008)

9. Steger C.: An Unbiased Detector of Curvilinear Structures. IEEE Computer Society.(1998) 